\title{
. Evolution of prudent predation in complex food webs: . mechanism and evidence
}

\author{
3 Orestes U. Gutierrez Al-Khudhairy ${ }^{1}$ and Axel G. Rossberg ${ }^{1}$ \\ ${ }_{4}{ }^{1}$ Queen Mary University of London
}

5 May 26, 2021

\begin{abstract}
6 Abstract
Prudent predators catch sufficient prey to sustain their populations but not as much as to undermine their populations' survival. The idea that predators evolve to be prudent has been dismissed in the 1970s, but the blunt arguments invoked 9 then are untenable in light of modern evolution theory. Evolution of prudent predation has repeatedly been demonstrated

in two-species predator-prey metacommunity models. However, the vigorous population fluctuations that these models predict are not widely observed. Here we show that in complex model food-webs prudent predation evolves by a different mechanism. We make testable predictions for empirical signatures of this mechanism and its outcomes. Then we discuss how these predictions are borne out across freshwater, marine, and terrestrial ecosystems. Demonstrating explanatory power of evolved prudent predation well beyond the question of predator-prey coexistence, the predicted signatures explain unexpected declines of invasive alien species, the shape of stock-recruitment relations of fish, and the clearance rates of pelagic consumers across the latitudinal gradient and 15 orders of magnitude in body mass. Specific research to further test and mobilise the utility of this theory is proposed.
\end{abstract}

\section{Hosted file}

GutierrezAl-Khudhairy2021Preprint_PrudentPredation.pdf available at https://authorea. com/users/414973/articles/522881-evolution-of-prudent-predation-in-complex-food-webs-

21 mechanism-and-evidence 